\title{
Quantification with normal limits: New cameras and low-dose imaging
}

\author{
Piotr J. Slomka, PhD, ${ }^{a, b}$ Mathieu Rubeaux, PhD, a and Guido Germano, PhD ${ }^{a, b}$ \\ a Department of Medicine, Cedars-Sinai Medical Center, Los Angeles \\ ${ }^{b}$ David Geffen School of Medicine, UCLA, Los Angeles
}

Received May 23, 2016; accepted May 23, 2016

doi: 10.1007/s12350-016-0563-7

\section{See related article, pp. 1626-1636}

\section{INTRODUCTION}

SPECT myocardial perfusion imaging (MPI) widely utilizes relative quantification of myocardial perfusion. This is accomplished by local comparisons of test patients to other scans of normal patients in most current quantitative SPECT MPI methods. ${ }^{1-4}$ These comparisons allow identification of local areas of hypoperfusion, typically in polar map coordinates. The set of normal patients is usually referred to as "normal database" or "normal limits" (when a collection of databases is considered, for example stress and rest databases). Relative quantification of myocardial perfusion with normal databases is a powerful clinical tool which has been documented to rival expert physicians' reading. ${ }^{5}$ Therefore, the recommendations regarding the appropriate use of such normal databases should be of great interest to all nuclear cardiology clinicians.

\section{NEW CAMERAS AND QUANTIFICATION OF PERFUSION}

Recently, new SPECT scanners with cadmium zinc telluride (CZT) crystals have been introduced together with novel scanner geometries, collimators, and sometimes upright patient positioning. ${ }^{6}$ In addition, most of these technologies utilize novel software reconstruction techniques incorporating various image

\footnotetext{
Reprint requests: Piotr J. Slomka, PhD, Department of Medicine, Cedars-Sinai Medical Center, Los Angeles; slomkap@cshs.org J Nucl Cardiol 2017;24:1637-40.

$1071-3581 / \$ 34.00$

Copyright (c) 2016 American Society of Nuclear Cardiology.
}

corrections, most notably resolution recovery. The regional count distribution of a normal SPECT MPI scan may significantly differ between these new scanners and conventional Anger cameras, and therefore new dedicated normal limits may be required. Indeed, normal distribution differences were observed in early studies using CZT cameras and separate specific limits have been recommended for these systems. ${ }^{7}$ For quantitative analysis of data obtained with CZT scanners, dedicated normal databases have been created, especially for the Discovery NM 530c

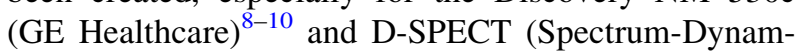
ics) ${ }^{7,11,12}$ cameras.

\section{NORMAL PERFUSION EVALUATED IN PHANTOM STUDY}

In this issue of Journal of Nuclear Cardiology, Zoccarato et $\mathrm{al}^{13}$ examine in detail the normal distribution of myocardial perfusion in seven different hardware systems (including conventional Anger cameras and CZT-based dedicated cardiac scanners) used to image the same cardiac phantom. Beyond the impact of the scanners employed, they also investigate the influence of the reconstruction methods and the count statistics on the uniformity of the activity in the myocardium. Whenever possible, the effect of attenuation correction is taken into account. Authors observe differences in the relative myocardial count distribution in the reconstructed images of the phantom between different systems and reconstruction methods. They conclude that different perfusion databases are indeed required for different scanners. However, they do not detect any significant differences between the databases when imaging time is decreased up to $25 \%$ of the reference time to simulate low-count statistics, and therefore they suggest that databases do not need to be modified for low-dose studies. 


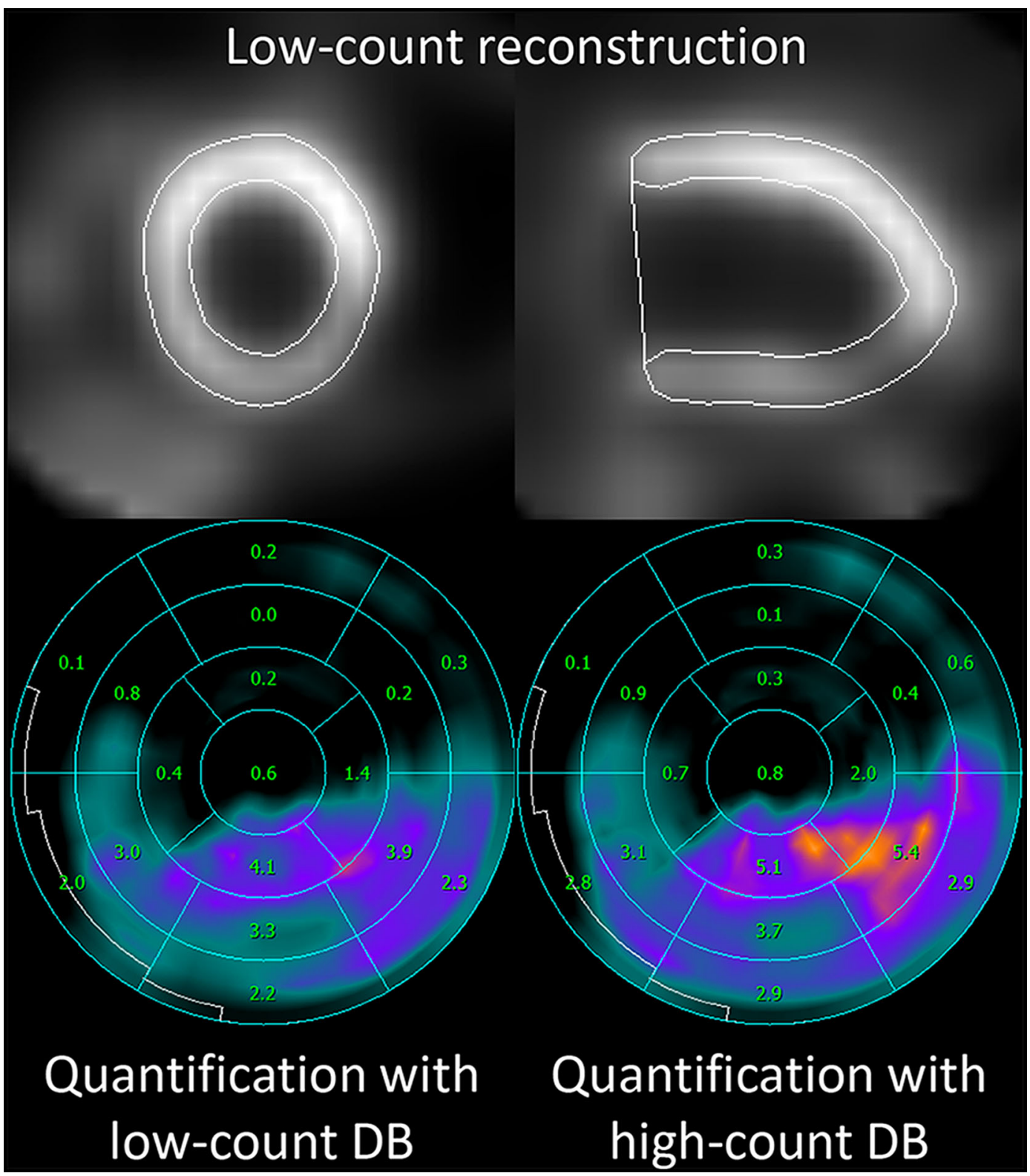

Figure 1. An example of low-count stress ${ }^{99 \mathrm{~m}} \mathrm{Tc}$ male scan with 1 million counts in the left ventricle (LV) (top) on the CZT camera and relative quantification of hypoperfusion severity (bottom) with low-count (bottom left) and high-count (bottom right) normal database (DB). These two databases are created from the same 15 normal male patients, but the low-count DB utilizes only $25 \%$ of the counts from the list-mode files ( $\sim 1$ million in each LV), compared to the highcount DB ( $\sim 4$ million in each LV). List-mode files allow reduction of counts used without repeating the image acquisition. ${ }^{14}$ Apparent increased hypoperfusion severity is seen due to lower inter-patient variation of the high-count DB. The defect extent was estimated in this patient as $21 \%$ with low-count DB and $28 \%$ with high-count DB.

\section{SHOULD WE STUDY PATIENTS OR PHANTOMS?}

To put in perspective the phantom results presented by Zoccarato et al, we can contrast it with some recent work by Nakazato et al evaluating the effect of count statistics on relative perfusion quantification in patients. ${ }^{14}$ In that study, the normal patients' list-mode raw data obtained from CZT SPECT scanner are reframed to simulate low-count images. They demonstrate that a progressive decrease of the left ventricular count levels leads to an increase of the segmental interpatient variation of the normal limits. Indeed, even though the relative count distribution for the low-count 
studies is similar to the high-dose studies, the interpatient variation is changing due to increased uncertainty. Consequently, Nakazato et al propose the use of count-specific normal limits to obtain comparable quantitative findings for studies with different count levels.

Thus, what could be the reason for this apparent discrepancy between the findings of phantom-based and patient-based studies of normal databases? The most likely answer lies in the definition of the "variability". In the study by Zoccarato et al, only one phantom dataset was collected for each camera and the count variability was thus defined as the coefficient of variation of counts in a given segment. However, when the actual normal limits are created in a clinical scenario, the variability is estimated from inter-patient differences after image normalization. This local variability measure is used in most clinical implementations of the relative quantification. $^{1-4}$ to derive the severity of the local perfusion decrease and consequently overall measures of hypoperfusion such as defect extent or perfusion deficit. Therefore, although a phantom study is a useful test for evaluation of the differences in relative perfusion distribution in the uniform myocardium related to the scanner hardware and reconstruction methods (abstracting it from patient or physiological differences), it is less applicable to testing the variability of the real-world inter-patient database.

\section{DO WE NEED LOW-COUNT DATABASES?}

Figure 1 shows an example of a low-count SPECT MPI (1 million counts in the left ventricle) from CZT camera and quantification of hypoperfusion severity performed with low-count and high-count normal databases. As seen in the bottom right of Figure 1, the use of the high-count database for low-count image results in an apparent overestimation of the defect size, since some variation considered within normal threshold by the low-count database could be considered belownormal threshold in the high-count database with tighter normal limits. Thus, the left ventricular count statistics of test patients should ideally be matched to the count statistics of the patients used to create the corresponding normal database. Nevertheless, if the variation increase is predictable, it would be possible to calibrate the highcount database for low-count imaging by slightly relaxing the confidence limits for what is considered a normal scan. Alternatively, it may be even acceptable to adjust the global normal threshold of defect extent or perfusion deficit upwards, when low-count studies are quantified with high-count databases. Ultimately, the overall diagnostic accuracy of low-count imaging has to be validated by comparison of receiver operating characteristics curves. Further studies are needed to truly evaluate the potential of normal limits to reducing the imaging dose and time in SPECT MPI.

\section{HOW MANY DATABASES DO WE NEED?}

The evidence in the studies to date indicates that dedicated normal limits are required for the optimal relative quantification when scanners and reconstruction methods differ significantly in design or if they use features such as resolution recovery or attenuation correction. To this end, most commercial implementations allow the creation of dedicated normal limits. On the other hand, generic databases for typical dual cameras and standard imaging protocols are likely to be applicable to any center utilizing such standard equipment and methods. With respect to low-count studies, we recommend to consider the photon count level in the left ventricle. If left ventricular counts are significantly lower than those in the studies used to create the database (due to lower injected dose or shorter imaging time) a new database or some recalibration of the final quantification results may be required. It should be noted however that low-dose studies that are acquired with proportional extension of the imaging time should present similar count level in the left ventricle and it is likely that the same database could be repurposed.

\section{CONCLUSIONS}

The selection of appropriate normal databases is of great importance for quantification of myocardial perfusion. The pattern of normal perfusion in the left ventricle depends on the scanner geometry, use of attenuation correction, and resolution recovery techniques. Therefore, dedicated normal databases may be required for the most optimal quantification of myocardial perfusion. Substantial reduction of ventricular counts may also necessitate updated database or recalibration of the quantitative results. Further definitive studies utilizing progressive list-mode reformatting and hard clinical endpoints are needed to probe the acceptable limits for the left ventricular count reduction.

\section{Acknowledgments}

This research was supported in part by Grant R01HL089765 from the National Heart, Lung, and Blood Institute/National Institute of Health (NHLBI/NIH) (PI: PS). Its contents are solely the responsibility of the authors and do not necessarily represent the official views of the NHLBI/NIH. 


\section{Disclosure}

Cedars-Sinai Medical Center receives royalties for the quantitative assessment of function, perfusion, and viability, and a minority portion is distributed to some of the authors of this manuscript (GG and $P S$ ).

\section{References}

1. Germano G, Kavanagh PB, Slomka PJ, Van Kriekinge SD, Pollard G, Berman DS. Quantitation in gated perfusion SPECT imaging: the Cedars-Sinai approach. J Nucl Cardiol. 2007;14:433-54.

2. Garcia EV, Faber TL, Cooke CD, Folks RD, Chen J, Santana C. The increasing role of quantification in clinical nuclear cardiology: the Emory approach. J Nucl Cardiol. 2007;14:420-32.

3. Ficaro EP, Lee BC, Kritzman JN, Corbett JR. Corridor4DM: the Michigan method for quantitative nuclear cardiology. J Nucl Cardiol. 2007;14:455-65.

4. Liu YH. Quantification of nuclear cardiac images: the Yale approach. J Nucl Cardiol. 2007;14:483-91.

5. Arsanjani R, Xu Y, Hayes SW, Fish M, Lemley M Jr, Gerlach J, Dorbala S, Berman DS, Germano G, Slomka P. Comparison of fully automated computer analysis and visual scoring for detection of coronary artery disease from myocardial perfusion SPECT in a large population. J Nucl Med. 2013;54:221-8.

6. Slomka PJ, Patton JA, Berman DS, Germano G. Advances in technical aspects of myocardial perfusion SPECT imaging. J Nucl Cardiol. 2009;16:255-76.

7. Sharir T, Slomka PJ, Hayes SW, DiCarli MF, Ziffer JA, Martin WH, Dickman D, Ben-Haim S, Berman DS. Multicenter trial of high-speed versus conventional single-photon emission computed tomography imaging: quantitative results of myocardial perfusion and left ventricular function. J Am Coll Cardiol. 2010;55:196574.

8. Duvall WL, Slomka PJ, Gerlach JR, Sweeny JM, Baber U, Croft LB, Guma KA, George T, Henzlova MJ. High-efficiency SPECT MPI: comparison of automated quantification, visual interpretation, and coronary angiography. J Nucl Cardiol. 2013;20:763-73.

9. Gregoire B, Pina-Jomir G, Bontemps L, Janier M and Scheiber C. The value of local normal limits in quantitative Thallium-201 CZT MPI SPECT. J Nucl Cardiol. 2016. doi:10.1007/s12350016-0430-6.

10. Nishiyama Y, Miyagawa M, Kawaguchi N, Nakamura M, Kido T, Kurata A, Kido T, Ogimoto A, Higaki J, Mochizuki T. Combined supine and prone myocardial perfusion single-photon emission computed tomography with a cadmium zinc telluride camera for detection of coronary artery disease. Circ J. 2014;78:1169-75.

11. Nakazato R, Tamarappoo BK, Kang X, Wolak A, Kite F, Hayes SW, Thomson LE, Friedman JD, Berman DS, Slomka PJ. Quantitative upright-supine high-speed SPECT myocardial perfusion imaging for detection of coronary artery disease: correlation with invasive coronary angiography. J Nucl Med. 2010;51:1724-31.

12. Nakazato R, Slomka PJ, Fish M, Schwartz RG, Hayes SW, Thomson LE, Friedman JD, Lemley M, Jr., Mackin ML, Peterson B, Schwartz AM, Doran JA, Germano G and Berman DS. Quantitative high-efficiency cadmium-zinc-telluride SPECT with dedicated parallel-hole collimation system in obese patients: Results of a multi-center study. J Nucl Cardiol. 2015;22:266-75.

13. Zoccarato O, Marcassa C, Leva L, Lucignani G, Savi A, Sciabbo C, Matheoud R, Lecchi M and Brambilla M. Difference in polarmap patterns using the novel technologies for myocardial perfusion imaging. J Nucl Cardiol. 2016. doi:10.1007/s12350016-0500-9.

14. Nakazato R, Berman D, Hayes M, Fish M, Padgett R, Xu Y, Lemley M, Baavour R, Roth N, Slomka P. Myocardial perfusion imaging with a solid state camera: Simulation of a very low dose imaging protocol. J Nucl Med. 2013;54:373-9. 